\title{
Ultrasound screening at 11-14 weeks of pregnancy for diagnosis of placenta accreta in mothers with a history of cesarean section
}

\author{
Ladan Younesi (1), Zahra Karimi Dehkordi (2), Zeinab Safarpour Lima (1), Ghazaleh Amjad (1)
}

(1) Shahid Akbarabadi Clinical Research Development Unit (ShACRDU), Iran University of Medical Sciences (IUMS), Tehran, Iran; (2) Department of Radiology, Shahid Akbar Abadi Hospital , Iran University of Medical Sciences, Tehran, Iran

This article is distributed under the terms of the Creative Commons Attribution Noncommercial License (CC BY-NC 4.0) which permits any noncommercial use, distribution, and reproduction in any medium, provided the original author(s) and source are credited.

\begin{abstract}
The aim of this study was to add ultrasonographic screening criteria for placenta accreta in patients with cesarean section history at 11-14 weeks of gestation in order to identify those with the high risk of placenta accreta. Consequently, using ultrasound follow-up and confirmation of diagnosis can reduce mortality and morbidity. Pregnant mothers who were referred for routine screening for ultrasonography at 11-14 weeks of gestation were enrolled in the study if they had a history of cesarean section. Of the 184 subjects who were included in the study, 23 of the low-risk groups were excluded from study for some reason such as miscarriage and a lack of required information on the time of delivery. Among the 152 subjects, 27 were classified in the high-risk group (17.7\%) and 125 subjects in the low-risk group $(82.3 \%)$. Only one case in the high risk group was identified in patients with placenta accreta by ultrasound and clinical diagnosis, which has undergone a cesarean section at the gestational age of 35 weeks. There was no relationship between placenta accreta and disease risk, placenta previa, cesarean section, maternal age, and gravidity.

Therefore, placenta accreta screening using ultrasonography can identify high-risk individuals at week 14-11 and with subsequent follow-up for improving prenatal prognosis..
\end{abstract}

Key Words: Placenta accreta, first trimester of pregnancy, history of cesarean section, ultrasound screening

Eur J Transl Myol 28 (4) $354-361,2018$

Placenta accreta is one of the life-threatening conditions in pregnancy resulting from the abnormal placental attachment to the myometrium, which leads to severe bleeding at time of attempted vaginal delivery. ${ }^{1-3}$ Placenta accreta is the main indication of emergency postpartum hysterectomy. ${ }^{4,5}$ Severe hemorrhage during the removal of the pairs (more than 2 litres or 4 pints) can lead to Disseminated Intravascular Coagulation (DIC), acute respiratory distress syndrome (ARDS), renal failure, and death. In most cases, emergency postpartum hysterectomy is mandatory and sometimes it is accompanied with more severe comorbidities such as cystostomy $(15.4 \%)$, ureteral injury $(2.1 \%)$, pulmonary embolism (2.1\%), and admission of patients to intensive care unit $(26.2 \%) .^{6-8}$ Placenta previa, previous cesarean section, and maternal age (mothers above 35 years of age) are the most important risk factors, and the first two are considered as independent risk factors for placenta accreta development. ${ }^{9,10}$ Other predisposing factors include prior uterine curettage, manual removal of the placenta, uterine malformation, prior uterine surgery (such as myomectomy), multiparity, uterine fibroids, and maternal sepsis. ${ }^{11}$ The incidence of placenta accreta increased in the past three decades, up to tenfold in the United States. Its rate is currently 1 in 2500 births, due to increased uterine surgery (curettage and cesarean section $)^{9,11}$ In another study in the United States, the incidence of placenta accreta have been quadrupled for the period of 1994 to 2002, the main cause being an increase in cesarean section rates. ${ }^{12}$ Precise prenatal diagnosis during pregnancy allows the surgeon to have an ideal performance with the required readiness and specific schedule, including surgical time, surgical site, availability of blood products, anesthesia and a skilled surgical team. All together they can decrease mortality and morbidity rates. ${ }^{13-15}$ 


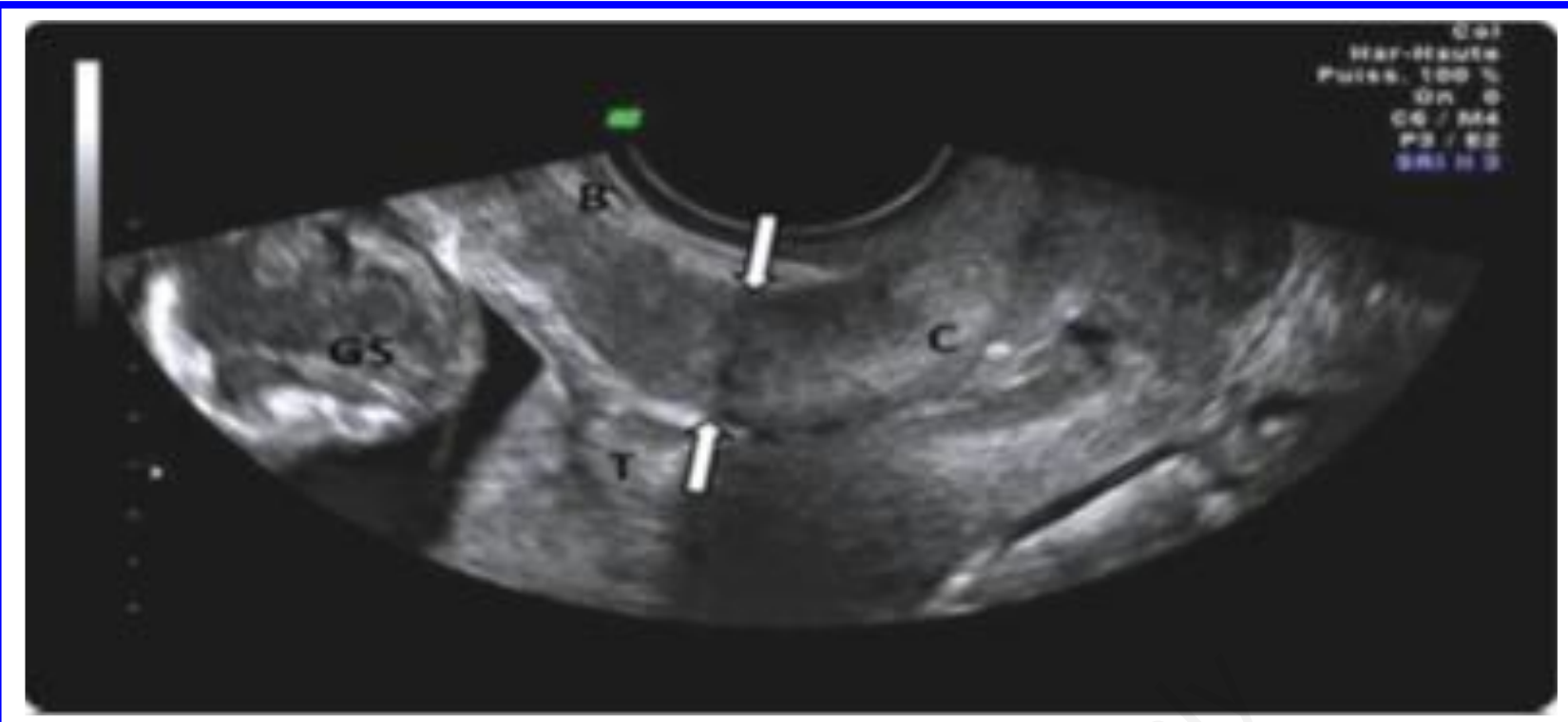

Fig 1. Transvaginal ultrasonography, midsagittal cross section in risky group: A low-lying placenta overlapping the scar, C: Cervix T: trophoblast (placenta) GS: gestational sac

Ultrasound is the elective diagnostic method for placenta accreta, that may detect this complication in $50-80 \%$ of cases. ${ }^{11,13,16}$ Despite attempts to detect placenta accreta in the second and third trimester of pregnancy, and the use of ultrasound and MRI, many cases of placenta accreta are diagnosed with pre and postpartum hemorrhages at the time of delivery. ${ }^{17,18}$ It is precisely hard to determine the exact incidence of placenta accreta, since the standard sources of diagnostic confirmation are histological findings. Currently, according to the Ministry of Health, the average cesarean section rate in Iran is three times more than the global average, and this trend increased from $35 \%$ of deliveries in 2000 to $40 \%$ in 2005 . This figure is up to $60 \%$ in private hospitals. According to the statistics provided in the year 2014 by the Ministry of Health, the incidence of cesarean delivery in some regions of Iran is up to $70 \%$ of births. Following an increase in the number of cesarean sections in the country, the complications of this surgery, such as placenta accreta and placenta previa, in subsiquent pregnancy would increase.

The main goal of our study was the addition of ultrasound criteria for screening of placenta accreta at 11-14 weeks of gestation in pregnant mothers with a history of cesarean section, in order to identify those at high risk for placenta accreta. Subsequent follow-up by ultrasound analyses and other diagnostic confirmations could reduce severe morbidity and mortality of this complication.

\section{Materials and Methods}

\section{Study population}

In this cohort study, the population consisted of pregnant women at 11 to 14 weeks of gestation [crown rump length $(\mathrm{CRL})$ of fetuse $=45-84 \mathrm{~mm}$, with a history of cesarean section, who were referred for routine first screening ultrasound (transvaginal sonography). Our study was conducted in Akbarabadi Hospital, Tehran, Iran, during a period of 2.5 years, from April 2012 to November 2014. Pregnant women entered the study if they had a history of at least one cesarean section. Informed consent was taken at enrollment for both entering study and for use of personal medical records. Demographic characteristics (age, number of pregnancies) and medical history (cesarean section, curettage, history of uterine surgery, etc.) were recorded in a questionnaire. In the transvaginal sonography, midsagittal plane includes the cervical and bladder canal and the lower part of the gestational sac. Ultimately, the patients were classified into two groups, a low-risk and a high-risk groups based on location of gestational ${ }^{1}$ sac, uterine scar and the relationship between the scar and the trophoblast (placenta). High risk group: the scar of the myometrium were inside the cavity (exposed scar) and above the lower edge of the placenta (the placenta is on the scar); moreover, the gestational sac also covered the cervix and the lower part of the uterus (the lower lying gestational sac) (Figure 1). Low risk group: if surgical scar wass protected by the cervical isthmic canal (Figure $2, \mathrm{~A})$ or the placenta was not placed on the innermost side of the cervical canal (no placenta Previa) and the placenta was located higher than the scar area (Figure 2,B). The high-risk group underwent a second ultrasound examination at 19- 31 weeks of gestation to 

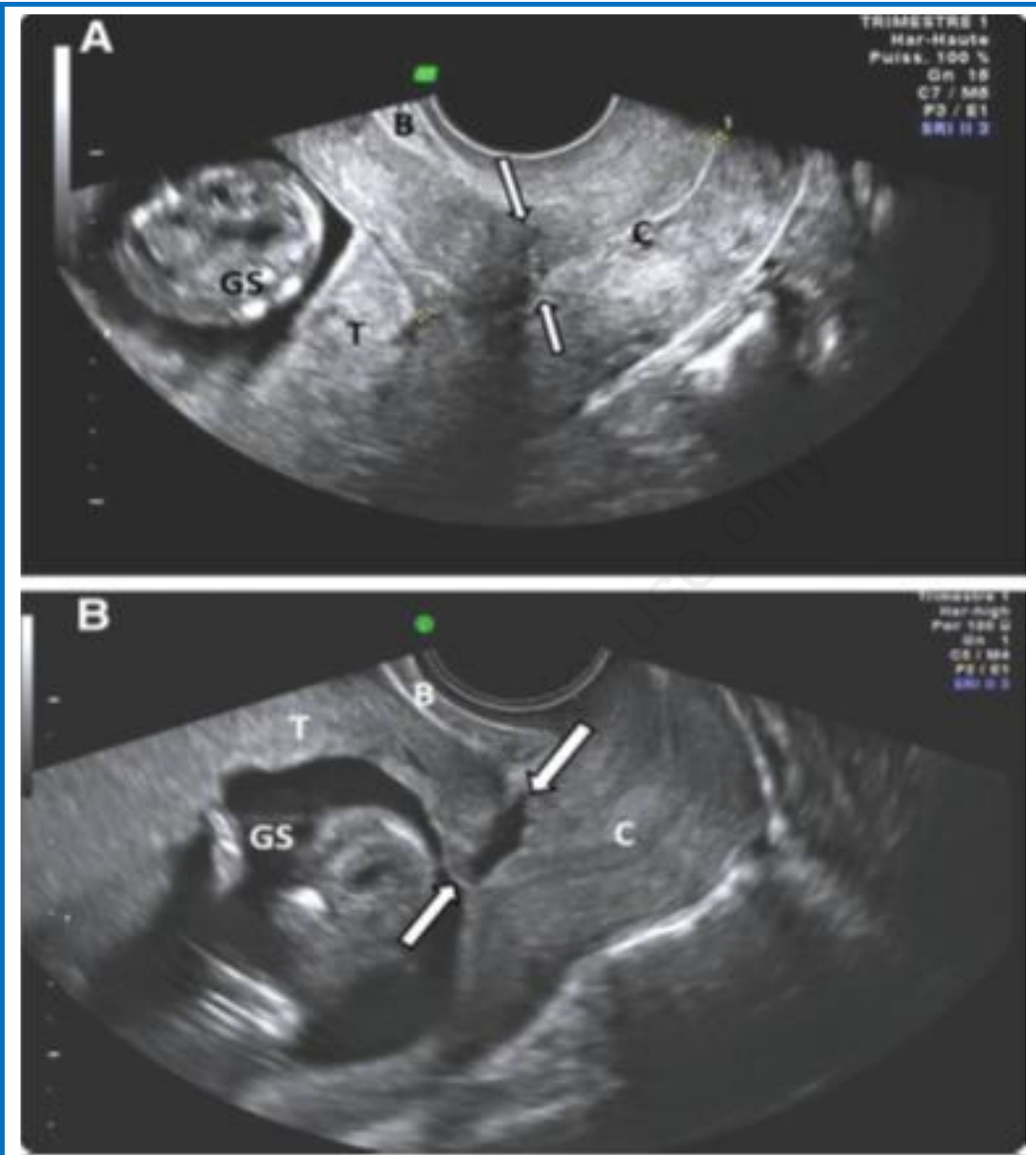

Fig 2. Transvaginal ultrasonography, midsagittal cross section, Low risk group, A: Protected scar and placenta at the edge of the endocervical canal; B: exposed scars was revealed but without overlapping of the placenta. C, Cervix; T, Throboplast (placenta); GS, gestational sac

confirm or exclude the presence of placenta accreta. Both groups were followed up to delivery and then the cases were recorded according to results of pathology or clinical diagnosis. The reference standard was considered based on the confirmation of histological findings. Although bleeding was sometimes controlled without the need for hysterectomy, if pathology data were not available, clinical findings included: 1. manual removal of the placenta was difficult or it needed a surgical approach; 2 . severe and uncontrollable bleeding after removal of the placenta in well-contracted uterus. Demographic data and medical history of mothers were 


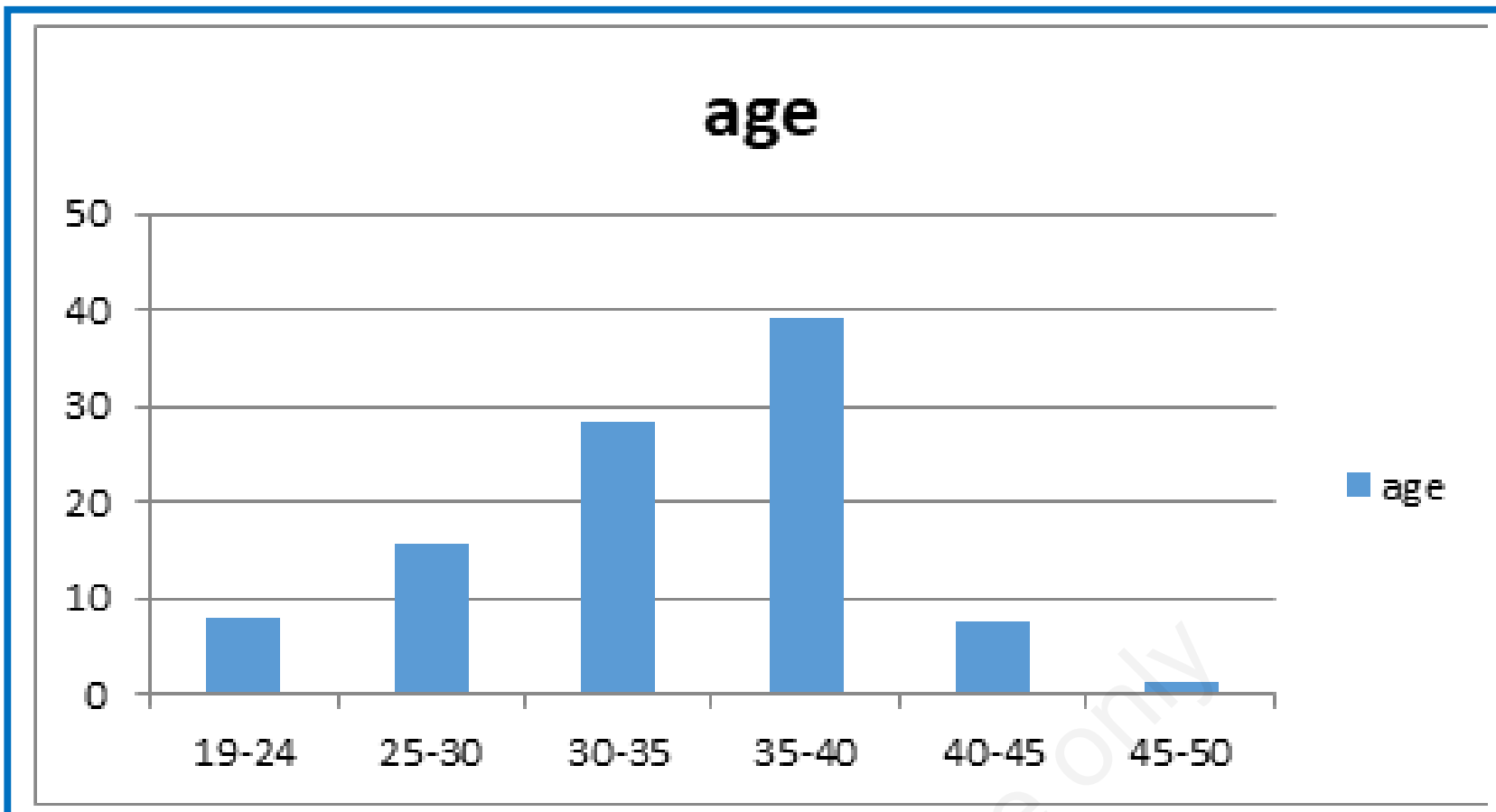

Fig 3. Frequency of subjects under study by age

recorded by ultrasound clerks in a questionnaire. Relationships between maternal demographic characteristics, such as maternal age, number of pregnancies, cesarean sections, history of curettage and myometectomy with placenta accreta, were evaluated by SPSS Statistics version 23. Ultrasound was performed by a radiologist expert in the fields of obstetrics and gynecology. Results were recorded in the patient's form, which included examining the location of the scars and placenta to determine the individual risk. All mothers were evaluated by the SIEMENS G 50 ultrasound machine with a vaginal probe of 9-4 MHz. Evaluation of postpartum pairing through pathologic outcomes (in the case of hysterectomy) or otherwise on clinical diagnosis proposed by a gynecologist.

\section{Sample size}

In order to determine the sample size, we needed an estimate of the prevalence of placenta accreta in Iran. Indeed, there have been no studies on the prevalence of placenta accreta, that is known to be directly related to the number of cesarean section. The rate of cesarean delivery in Iran is three times more than the one reported for global statistics. Thus, the prevalence of placenta accreta in Iran will be different from countries like the United States. While almost all litterature results were case study, just one was similar to our study, but only 105 persons were screened for sonography. Thus, we set the sample size of our study to 200 subjects.

\section{Data analysis}

SPSS 23 software was employed in the current study and all data were analyzed by chi-squared test. P-value of less than 0.05 was considered to be statistically significant.

\section{Results}

Of the 184 subjects enrolled in the study, 23 of the lowrisk groups were excluded, of which three were excluded due to abortion for one to six weeks after ultrasound and others due to a lack of required information on time of delivery. Furthermore, 9 subjects in the high risk group were excluded, one person due to abortion at 14 week of pregnancy and 8 others due to the lack of follow-up sonography. Among the remaining 152 subjects, 27 were classified in to the high risk group $(17.7 \%)$ and 125 in the low risk group (82.3\%).

In people with low risk, no placenta accreta cases were recorded after childbirth follow up. Among the 27 high risk subjects, in the early phase (11-14 weeks), eight (30\%) showed a placenta previa (partial or complete). Among some persons in the high risk group, there were no other ultrasound criteria such as placenta previa or low lying, while overlapping between placenta and scars was lost in follow-up ultrasounds $(66.6 \%$ at 18 weeks, $22.2 \%$ at 22 weeks and $3 \%$ at 30 weeks pregnancy). In addition, follow-up ultrasounds in these subjects did not show evidence of a placenta accreta (such as placental lacunae or retroplacental complex). Only two subjects in the high risk group, which had an ultrasound examination before the end of the study, were diagnosed with placenta accreta. The first case was 38 years old, with a gravidity of 4 , who had a history of 3 times 
cesarean section, one of which was an emergency cesarean section, and also had a history of curettage. The person referred to the center at 11 weeks of gestation and placenta previa was reveled in the ultrasound and scar was also exposed. Other evidence was also found in the first ultrasound, including a $5 \mathrm{~mm}$ suspected defect area in the retroplacental complex near the inner part of cervical sphincter.

Two placental venous lakes were observed in 11 and 12 $\mathrm{mm}$ diameters in placenta, while placenta previa was observed in the subsequent ultrasounds. The size of the lacunae increased to $40 \mathrm{~mm}$ at pregnancy week 18 and $70 \mathrm{~mm}$ at pregnancy week 26. Moreover, myometrial thinning was observed at the site of the defect at pregnancy week 26 , while a cystic spaces filled with blood was observed in the posterior myometrial adjacent to the defect at week 30 . The serous level of the uterus and bladder were normal in the last ultrasound of the patient at 34 weeks.

The patient with ultrasound diagnosis of placenta accreta was monitored, when it was found to be approximately $30 \mathrm{~mm}$ in length. Elective caesarean section was performed by a team of gynecologic surgery, general surgery and anesthesiologist at the age of 35 weeks. During surgery, placenta previa was confirmed. It was not manually detachable, but was surgically removed and a subsequently compression suture was performed. It is worth noting that suture was sufficient to control bleeding, so that and did hysterectomy was not required. Unfortunately, pathology diagnosis was not possible because of the fragmentation of the placenta during its removal and lack of hysterectomy.

The second case was a 30-year-old woman with gravidity 3 and a history of emergency cesarean section due to placenta previa who had been exposed to a scar at 13 weeks of pregnancy and overlapped with a low lying placenta. The placenta remained low lying and myometrial hyperemia and suspected retroplacental insufficiency were observed in the area attached to the scar following susequent ultrasonography, although no evidence was found for formation of lacunae. The patient was subjected to cesarean section at 38 weeks, The placenta was in lower part and was not easily detached when the surgeon attempted to manually separate the placenta. During the cesarean section, the patient suffered from severe hemorrhage, therefore she was admitted to the ICU for two days and received two units of packed red blood cell. In addition to the mild adhesion of the placenta, the uterus of the patient was also atonic, the bleeding could not be solely due to the placenta accreta as a definitive clinical diagnosis. Therefore this case was not included in the list of diagnosis of placenta accreta. The highest age range of subjects was determined as 40-35 (39\%), followed by a range of $30-35$ (28.3\%). Age-related frequency distribution of the subjects is shown in Figure 3. Among all enrolled subjects, history of curettage was not found in $67 \%$ of the subjects; in the remaining $25 \%$ of samples, history of curettage was obtained (Table 1). Of the 152 subjects, 136 had once a cesarean section $(73.5 \%)$, (Table 2). Among the subjects, gravidity 2 was detected in $101(52.2 \%)$ and 58(31.1\%) of the patients seen in other gravidity, gravidity 3 (Table 3 ). Among all enrolled subjects, 44 (24\%) patients had an emergency cesarean section, and history of myomectomy was seen in 3 patients $(1.6 \%)$. Based on the chi square test, a significant correlation was found between the placenta accreta and risk of disease $(p=0.048)$. This means that the occurrence of placenta accreta is higher in the high risk group as compared to the other group. Based on chi square test, there was no significant correlation between placenta accreta and gravidity $(\mathrm{p}=0.078)$. Furthermore, a significant relationship was found between the placenta previa and placenta accreta, which means that the presence of placenta previa is associated with an increase in the incidence of placenta accreta. We have consistently indicated a statistically significant association between placenta accreta and number of previous cesarean sections $(\mathrm{p}<0.001)$. As matter of fact, an increase in the number of previous cesarean sections was associated with higher risk of placenta accreta. Based on the information provided herein, the occurrence of eccentricity was not significantly related to history of uterine surgery (myomectomy or surgery for uterine anomalies), history of curettage, age of the patient, gravidity and history of emergency cesarean section.

\section{Discussion}

Placenta accreta spectrum is a complex disorder related to abnormal adherence of the placental trophoblast to the uterine myometrium which leads to severe hemorrhage at the time of attempted vaginal delivery. It is considered as an important pregnancy condition strongly linked to abnormal placenta growth into the uterus wall. ${ }^{19}$ In this study, there was a significant correlation between the number of previous cesarean section and placenta accreta. Moreover, there was a strong relationship between placenta previa and placenta accreta. In the current study, no significant correlation was found between placenta accreta and other factors such as history of curettage, emergency cesarean section, maternal age, gravidity and uterine surgery. In the present study, the incidence of placenta accreta was $0.65 \%$ among those with a history of cesarean section, or 65 per 10,000 women with a history of cesarean section. Given the fact that the population studied is merely mothers with a history of cesarean section, the overall prevalence of Placenta accreta cannot be estimated based on the type of delivery. Prevalence of placenta accreta in women with a history of cesarean section and placenta previa (in the preliminary study) was calculated to be $12.5 \%$. Based on data presented, there was significant relationship between placenta accreta and risk of disease, which is more likely to be 


\section{Ultrasound screening for placenta accreta diagnosis}

Eur J Transl Myol 28 (4): 354-361, 2018

higher in high-risk individuals than in the other group due to the factors that were considered for the high risk group such as placenta previa. Many studies have also reported a significant relationship between placenta previa and placenta accreta, that is an independent risk factor, and also the presence of exposed scar with overlapping trophoblast is another known risk factor for
Furthermore, our findings demonstrated the association of placenta accreta with the number of previous cesareans and placenta previa, that an increase in the number of cesarean sections is likely linked to the probability of placenta accreta, this association has also been proven in other studies. ${ }^{3}$ As other study indicated, in patients with placenta previa, placenta accreta risk

Table 1. History of curettage and its frequency in the study population

\begin{tabular}{lll}
\hline Number of Curettage & Frequency & Frequency percentage \\
\hline 0 & 124 & 67 \\
1 & 45 & 25 \\
2 & 13 & 7 \\
3 and more & 1 & $0 / 5$
\end{tabular}

$* * * * * * * * * * * * * * * * * * * *$

Table 2. Distribution of previous cesarean section in the population under study

\begin{tabular}{lll}
\hline Number of Previous cesarean & Frequency & Frequency percentage \\
\hline 1 & 136 & $73 / 5$ \\
2 & 43 & $23 / 5$ \\
3 & 5 & 3 \\
4 and more & 0 & 0 \\
\hline
\end{tabular}

$* * * * * * * * * * * * * * * * * * * * * * *$

Table 3. The frequency of pregnancy in the study population

\begin{tabular}{lcc}
\hline Number of pregnancy (gravidity) & Frequency & Frequency percentage \\
\hline 2 & 101 & $55 / 2$ \\
3 & 58 & $31 / 1$ \\
4 & 23 & $12 / 9$ \\
5and more & 2 & $0 / 8$ \\
\hline
\end{tabular}

placenta accreta.

The possible mechanism mentioned at the site of the scar may be the cause of placenta accreta in this site. has been found to be $2 \%$ in subjects less than 35 years old with no history of cesarean section, while this risk increased to $39 \%$ in subjects over the age of 35 with 
history of two or more cesarean sections. ${ }^{16}$ It is worth noting that placenta previa is one of the important risk factors for placenta accreta formation, those who have a history of cesarean section and placenta previa in ultrasound screening are considered to be at high risk for placenta accreta, and follow-up sonography is recommended. This shows the independence of factors such as maternal age, cesarean section and placenta previa in the occurance of placenta accreta. Another study showed that in patients with placenta previa, the risk of placenta accreta was $3 \%$ in women with no previous cesarean section, while in women with one cesarean section the risk of disease was $11 \%$. Based on the data presented in studies, placenta accreta risk varied from $40 \%$ in patients with two previous cesarean sections to $69 \%$ in subjects with three or more cesarean sections ${ }^{3-5}$. A significant relationship between placenta accreta with history of curettage, emergency cesarean section, patient's age and gravidity were not established in our study. The results of other studies are contrary to our findings. Almost all studies have shown age association with placenta accreta and is known as an independent risk factor for placenta accreta, where the age of 35 years has been considered as a risk age in many studies. ${ }^{9-11}$ In some studies, there has been no relationship between placenta accreta, history of curettage and emergency cesarean section ${ }^{9-11}$,but other studies described these variables as risk factors of placenta accreta. ${ }^{9-11}$ Only one case of placenta accreta was present in our study. We need to consider all of aforementioned findings in light of low sample size and low number of confirmed cases with placenta accreta. The relationship of placenta accreta with myomectomy and surgery for uterine anomalies was not observed in our study, which could be due to low sample size and low uterine surgeries in the population under study. As previous studies indicated, there have been a significant correlation between surgery on the uterus and placenta accreta, which was justified by a similar mechanism of $\mathrm{C}$-sections scars (defecation in decidua basalis and endometrial injury). Due to the very low prevalence of placenta accreta and the presence of a case of placenta accreta in this study, the evaluation of the relationship between the disease and the assessed factors requires a study with a larger sample size. In an early assessment of people in terms of the probability of placenta accreta, subsequent evaluation of high-risk individuals can be more sensitive; therefore, based on this study and other previous studies, we recommend that pregnant mothers with a history of cesarean section be screened for site of placentation and c-section scar and follow-up ultrasound should be performed. Regarding the follow-up process of the high-risk group in the study, the high-risk ultrasound findings in terms of placenta accreta were eliminated at screening time in $88.8 \%$ of cases, up to 22 weeks $(66.6 \%$ at 18 weeks and $22.2 \%$ at 22 week), where other evidences of placenta accreta ultrasound such as retroplacental complex, and lacunae were not observed, it is recommended that high-risk individuals perform a follow-up ultrasound at 22-2 weeks. Many cases with placenta accreta have been retrospectively diagnosed in the first and second trimester of pregnancy and have been also detected with severe bleeding after dilatation and curettage, which led to emergency hysterectomy in many cases. Evaluation in early stages of people at risk of placenta accreta is valuable. In addition, after an early evaluation of people in terms of the probability of placenta accreta, subsequent evaluation of high-risk individuals will be more important. In conclusion, we recommend that mothers with a history of cesarean section need further evaluation for site of placentation and c-section scar. Regarding to the insufficient sample size due to low prevalence of placenta accreta in this study, another study with higher observations is recommended to evaluate the factors affecting placenta accreta. On the other hand, in order to estimate the actual prevalence of placenta accreta in Iran, a comprehensive study should be conducted in light of all types of pregnancy in a wide range of time.

\section{List of acronyms}

ARDS - acute respiratory distress syndrome

c-section - cesarean section

CRL - crown rump length

DIC - disseminated intravascular coagulation

ICU - Intensive care unit

NT - nuchal thickness.

NB - nasal bone

\section{Author's contributions}

LY, ZKD, ZS, and GHA equally participated in experimental design, data collection, writing and revision of the manuscript.

\section{Acknowledgments \\ Funding: None.}

\section{Conflict of Interest}

The authors declare no conflicts of interests.

\section{Ethical Publication Statement}

We confirm that we have read the Journal's position on issues involved in ethical publication and affirm that this report is consistent with those guidelines.

\section{Corresponding Author}

Zahra Karimi Dehkordi , Department of Radiology, Shahid Akbar Abadi Hospital, Iran University of Medical Sciences, Tehran, Iran. Tel: 00989133823296

Email: Z_k_dehkordi@yahoo.com

E-mail of co-authors

Ladan Younesi: younesi.l@iums.ac.ir

Zeinab Safarpour Lima: safarpourlima.z@iums.ac.ir Ghazaleh Amjad: Amjad.gh@iums.ac.ir 


\section{Ultrasound screening for placenta accreta diagnosis}

Eur J Transl Myol 28 (4): 354-361, 2018

\section{References}

1. Oyelese Y, Smulian JC. Placenta previa, placenta accreta, and vasa previa. Obstet Gynecol 2006;107:927-41.

2. Silver RM, Landon MB, Rouse DJ, et al. Maternal morbidity associated with multiple repeat cesarean deliveries. Obstet Gynecol 2006;107:1226-32.

3. Clark SL, Belfort MA, Dildy GA, et al. Maternal death in the 21st century: causes, prevention, and relationship to cesarean delivery. Am J Obstet Gynecol 2008;199:36.e1-5.

4. Shellhaas CS, Gilbert S, Landon MB, et al. The frequency and complication rates of hysterectomy accompanying cesarean delivery. Obstet Gynecol 2009;114:224-9.

5. Flood KM, Said S, Geary M, Robson M, Fitzpatrickpartum hysterectomy over the last 4 decades. Am J Obstet Gynecol 2009;200:632.e16.

6. Silver RM, Landon MB, Rouse DJ, et al. Maternal morbidity associated with multiple repeat cesarean deliveries. Obstet Gynecol 2006;107:1226-32.

7. Usta IM, Hobeika EM, Musa AA, et al. Placenta previa-accreta: risk factors and complications. Am J Obstet Gynecol 2005;193:1045-9.

8. Lau WC, Fung HY, Rogers MS. Ten years experience of caesarean and postpartum hysterectomy in a teaching hospital in Hong Kong. Eur J Obstet Gynecol Reprod Biol 1997;74:133-7.

9. Miller DA, Chollet JA, Goodwin TM. Clinical risk factors for placenta previa-placenta accreta. Am J Obstet Gynecol 1997; 177:210-4

10. Clark SL, Koonings PP, Phelan JP. Placenta previa/ accreta and prior cesarean section. Obstet Gynecol 1985;66:89.

11. Warshak CR, Eskander R, Hull AD, et al. Accuracy of ultrasonography and magnetic resonance imaging in the diagnosis of placenta accreta. Obstet Gynecol 2006;108:573-81.

12. Wu S, Kocherginsky M, Hibbard JU. Abnormal placentation: twenty-year analysis. Am J Obstet Gynecol 2005;192:1458-61.

13. Usta IM, Hobeika EM, Musa AAA, et al. Placenta previa-accreta: risk factors and complications. Am J Obstet Gynecol 2005;193:1045-9.

14. Briery CM, Rose $\mathrm{CH}$, Hudson WT, et al. Planned vs emergent cesarean hysterectomy. Am J Obstet Gynecol 2007;197:154.e1-5.

15. Kayem G, Davy C, Goffinet F, et al. Conservative versus extirpative management in cases of placenta accreta. Obstet Gynecol 2004;104:531-6.

16. Comstock $\mathrm{CH}$. Antenatal diagnosis of placenta accreta: a review. Ultrasound Obstet Gynecol 2005;26: 89-96.

17. Eller AG, Porter TF, Soisson P, Silver RM. Optimal management strategies for placenta accreta. BJOG 2009;116:648-54.

18. Eller AG, Bennett MA, Sharshiner M, et al. Maternal morbidity in cases of placenta accreta managed by a multidisciplinary care team compared with standard obstetric care. Obstet Gynecol 2011;117:331-7.

19. Bartels HC, Postle JD, Downey P, Brennan DJ. Placenta Accreta Spectrum: A Review of Pathology, Molecular Biology, and Biomarkers. Dis Markers 2018 Jul 3;2018:1507674. doi: 10.1155/2018/1507674. eCollection 2018.

Received for publication: $15 / 8 / 2018$

Revision received: 03/9/2018

Accepted for publication: 03/9/2018 